\title{
APUNTES PARA UNA ANTROPOLOGÍA DEL ESPACIO. Consideraciones desde la geografía clásica a la geografía cultural
}

\author{
Isaura Cecilia GARCÍA LÓPEZ \\ Benemérita Universidad Autónoma de Puebla (México). \\ isaurac_2310@yahoo.es
}

\begin{abstract}
NOTES FOR AN ANTHROPOLOGY OF SPACE. Considerations from classical geography cultural geography
\end{abstract}

Resumen: Actualmente se ha desarrollado un campo de la antropología que dedica sus esfuerzos al estudio del espacio, el presente es una contribución a la teoría antropológica que se fundamenta en los aportes de la geografía vinculados con la sociología y la antropología, asimismo reconocer las propuestas de diversos autores, en la tradición clásica alemana y francesa, quienes han señalado que la reflexión teórica debe partir de la relación entre el espacio y la sociedad, retomando los importantes aportes de la escuela posibilista de Vidal de la Blache en Francia, de la antropogeografía de Ratzel, la sociología urbana R. Park, H. Lefevbre y otros, que buscaron explicar el comportamiento espacial de las sociedades modernas. Para lo cual se propone el término de espacio funcional antropógeno, obtenido con base en la reconstrucción teórica de nociones como espacio, paisaje, territorio, región y lugar, desde una perspectiva sociocultural.

Abstract: Currently, it's been developed a field of anthropology that focus in the study of space, the present contribution at the theory anthropology, what base in the geography studies related to sociology and anthropology, at the same time that identifying different authors proposals, in the classic tradition German and French, who have pointed out that the theoretical reflection between the space and the society, retaken the important contributions of the Posibilista school. Of Vidal de la Blanche in France, the anthropogeography of Ratzel, urban sociology R. Park, H. Lefebvre and others, who sought to explain the spatial behavior of modern societies? For which the term anthropogenic functional space obtained based on the theoretical reconstruction of notions such as space, landscape, territory, region and place, from a socialcultural perspective is proposed.

Palabras clave: Espacio. Territorio. Lugar. Antropología del espacio. Urbano

Space. Territory. Location. Anthropology of space. Urban 


\section{Lo más grande es el espacio, porque lo encierra todo.}

Parte fundamental de la antropología del espacio radica en comprender la relación que los sujetos construyen con su entorno, razón por la cual subrayamos la importancia que tiene el vínculo entre la geografía y la antropología, particularmente en relación con la organización territorial; retomando las ideas de Robert Ezra Park, para afirmar que "las relaciones sociales están ampliamente determinadas por las distancias físicas y la estabilidad social queda asegurada, cuando los seres humanos se desarrolló una metodología:” (Park, 1999).

Enunciado que motivó el cuestionamiento, en qué medida las relaciones sociales están determinadas por el ámbito geográfico, y el valor simbólico que les otorga el sujeto, objeto de este escrito. Si bien hoy día esas distancias están disminuidas por la comunicación y la informática, consideramos que aún el sujeto sigue apropiándose de su espacio, marcando, dejando huella en su territorialidad.

Los estudios sobre el espacio ${ }^{1}$, se situaron desde la geografía clásica y su vinculación con la sociología, iniciaron como investigaciones que propugnaban el estudio de las relaciones entre los procesos sociales y las transformaciones espaciales, hacia finales del siglo diecinueve y veinte.

Para la comprensión de que el objeto de estudio de la geografía es el espacio, se reconocieron las propuestas de diversos autores, en la tradición clásica alemana y francesa, quienes han señalado que la reflexión teórica debe partir de la relación entre el espacio y la sociedad. Amén de la debilidad manifestada por los geógrafos de la época, para explicar el comportamiento espacial en las sociedades modernas.

\section{De la Geografía Física a la Geografía Humana}

Desde sus orígenes, la geografía significó el estudio de la tierra y de la relación entre la naturaleza y el hombre. También surgieron interrogaciones acerca de cómo el hombre es el que transforma la naturaleza, provocando diversos procesos en favor de su hábitat, al mismo tiempo generando contaminación, deterioro, agotamiento y daño a su entorno. Desde estas y otras interrogantes resulta la geografía, cuyo objeto de estudio fue el análisis del hombre y su relación con la naturaleza; para su estudio inicialmente se dividió en geografía física, y geografía humana.

En esta exploración se encontró que, los geógrafos del siglo XIX, plantearon la pregunta acerca de cómo el paisaje ha influido en los grupos humanos, las sociedades y en sus relaciones sociales; indagación que propició la necesidad de una geografía humana, misma que el geógrafo alemán Ratzel nombró Antropogeografía, entendida como una geografía social que contempla la geografía humana y regional. Está propuesta, generó el llamado determinismo geográfico, más la noción de paisaje ${ }^{2}$, sumada a los conceptos de área y región cultural. Nociones epistemológicas que definieron el objeto de estudio de la geografía, con ello nacieron otros conceptos como: lugar y territorio. ${ }^{3}$

Las indagatorias y discusiones continuaron entre la antropogeografía y la sociografía, por más de cien años, siempre con la intención de determinar el objeto de estudio de la

1 Entendemos que las primeras nociones acerca del espacio, lo identifican con una porción específica de en el sentido de espacio geográfico.

2 Entendido como medio ambiente, en un sentido amplio.(Luis Gómez, 1983).

3 La discusión de estos conceptos clave en la geografía, ha generado debates epistemológicos entre los geógrafos y los no geógrafos, sociólogos o antropólogos. Revisar: Geografía Crítica, Cuadernos críticos de Geografía Humana, España: Universidad de Barcelona, que publica en diversos números los debates al respecto. http:// www.ub.edu/geocrit 
geografía, la morfología del paisaje cultural, o la relación del hombre con su ambiente, denominada sociografía a la cual Hassinger determinó como estudio globalizador de las comunidades humanas en su relación con el paisaje (Luis Gómez, 1983).

Según Hassinger en 1919, la geografía del paisaje, como ninguna otra ciencia contaba con un objeto propio, "distingue las múltiples relaciones que se establecen entre las manifestaciones de la naturaleza viva y la no viva, con las personas de diferentes estratos sociales"(Morlains, 2005), precisión vital para la antropología del espacio.

Igualmente importante fue la propuesta de la geografía cultural, derivada de la historia que ya desde Hipócrates señalaba el impacto del ambiente sobre la sociedad. De estas discusiones se generaron las propuestas de los geógrafos alemanes, holandeses y franceses; entre la geografía general y la regional, geografía humana o geografía social, discusiones que perviven en la contemporaneidad. Señalaremos en principio la polémica entre el determinismo alemán y la escuela posibilista de Vidal de la Blache en Francia. Una de las dificultades más recurrentes fue la de no poder explicar fácilmente cómo se da la organización del espacio en las sociedades modernas.

\section{Antropogeografía}

Ratzel, geógrafo evolucionista estudioso de la etnografía, formuló el término antropogeografía, considerado por diversos autores como sinónimos de geografía humana y geografía cultural, concepto que integra la mirada interdisciplinaria dentro del ámbito de las ciencias sociales. Donde, el espacio es entendido como necesario e indispensable para la vida del hombre, englobando al trabajo con la naturaleza o la sociedad, en la lucha del dominio por el espacio. Esta propuesta desarrolló dos conceptos clave: "territorio y espacio vital"; el primer término lo comprende como la apropiación de una parte del espacio (superficie territorial). por determinado grupo humano; mientras que el segundo, es el reflejo la representación de los requerimientos territoriales de una sociedad en función de su desenvolvimiento tecnológico, del total de la población y los recursos naturales (Morlains, 2005).

Muy criticado, el determinismo geográfico, se completó con la conceptualización propuesta por Hartshorne quien planteó el concepto de espacio absoluto como: "el conjunto de puntos que tienen existencia en sí, siendo independiente de cualquier cosa" (Lobato Correa, 1998). ${ }^{4}$

De gran utilidad para la antropología es cuando Hartshorne asume que el espacio y el tiempo asocian todas las dimensiones de la vida; a más de que constituyó a la geografía como el estudio de los fenómenos organizados de modo espacial y a la historia como la estudiosa de los mismos en su dimensión temporal. Lo cual le permitió utilizar el concepto de espacio, como un área en sí misma (combinación única de fenómenos naturales y sociales). Se subraya que ha sido histórica la conflictividad que ha tenido la geografía para la comprensión de los fenómenos sociales; si bien, la antropogeografía fue entendida como la "ciencia de la relaciones", cuyo interés era la comprensión del predominio de la naturaleza sobre los seres humanos, reconociendo la importancia de explicar los fenómenos más allá de sólo describirlos.

A las anteriores propuestas, siguió el 'posibilismo', cuyo planteamiento teórico más relevante fue la idea de que el medio ambiente modela a las culturas humanas, consideración

4 Confrontar, definición de Harstone: "El espacio está dotado de una serie de propiedades específicas, independiente de los fenómenos. Se trata de un espacio absoluto que aparece como un receptáculo que contienen cosas. Influido por la visión ideográfica define cada área como una peculiar combinación de elementos físicos y humanos que han sido modelados históricamente y que están dotados de ciertos atributos (tamaño, fisonomía, cultura etc.). que le otorgan una personalidad propia y diferenciada. Junto a los atributos materiales, señala la existencia de una comunidad cultural, de un sentimiento colectivo de pertenencia" (Delgado Mahecha, 2003). 
que marcaba los límites del desarrollo cultural, en la frontera de lo "posible". 5

A decir de otros autores, en relación con el espacio absoluto y relativo, como Daniel Harvey, esta conceptualización permitió argumentar que para comprender los fenómenos urbanos, se tendría que reconocer que existen tantos conceptos de espacio como diversas son las prácticas humanas, concepción con la que concordamos:

"En adelante, el espacio no es en sí mismo ni absoluto, ni relativo, ni relacional, pero puede llegar a ser una de estas cosas o toda a la vez según las circunstancias. El problema de una nítida conceptualización del espacio se resuelve a través de la práctica humana con respecto a él. Dicho de otro modo, no existe respuestas filosóficas a las preguntas filosóficas que surgen acerca de la naturaleza del espacio, sino que las respuestas residen en la práctica humana" (Harvey, 1977: 5-6).

Más adelante, este autor advierte que para el espacio la variable más importante era la distancia, entendida como la que determina la diferenciación espacial en un espacio homogéneo, de la cual derivó la noción de espacio relativo, el cual: "se entiende a partir de las relaciones entre los objetos, relaciones que implican costos -dinero, tiempo, energía"(Lobato Correa, 1998a: 26).

Aunque el autor asegura que el espacio no es absoluto, ni relativo, ni relacional, puede ser una de estas, o todas a la vez según las circunstancias. Para su estudio se requiere tanto de la geografía como de la sociología, en tanto se apoyen en la perspectiva de la percepción y de la lingüística del sujeto, partiendo esencialmente de la práctica humana.

Para fundamentar esta relación Harvey propone utilizar la clasificación elaborada por el filósofo Cassirer en 1944, elaborada con base en la experiencia: espacio orgánico, espacio perceptual y espacio simbólico. Considera, al primero como la transmisión genética, desde la perspectiva de la biológica y etología; la segunda más compleja, comprende la experiencia sensitiva, táctil, olfativa, acústica, cinestésica; este tipo espacial concibe el esquema o mapa mental como necesario además de integrar por la práctica a la memoria, relacionada con el modo de pensamiento cultural adquirido, el tipo espacio simbólico se determina a partir de la experiencia espacial abstracta, permite la interpretación y representación simbólica (Harvey, 1979: 22).

Conceptualización también observada en las propuestas teóricas de H. Lefebvre, quien argumentó, en Espacio y política, la posibilidad de que el espacio "desempeñe un papel o una función decisiva, en la estructuración de una totalidad, de una lógica, de un sistema", aunque no se le puede derivar de este, asimismo afirmó que "muy por el contrario se debe poner de manifiesto su función (práctica y estratégica)" (Lefebvre, 1976).

En este sentido subrayó que no se trata sólo de localizar (una necesidad o función)., sino de espacializar una actividad social vinculada a su conjunto, produciendo un espacio apropiado, noción analizada páginas adelante $(I b .: 9)$..

\section{Posibilismo}

Con este término se reconoce la propuesta francesa al respecto del espacio. La concepción global de la geografía emanada de las propuestas francesas, que separaron a la geografía humana de la geografía física, derivaron en la necesidad de generar "estudios regionales para comprender la diversidad de ambientes y su influencia sobre los hombres que viven en él" (Berdoulay, 1983).

5 La discusión, en términos antropológicos continúo hacia la ecología humana y la diversidad cultural, en este trabajo seguiremos la discusión sobre el espacio, líneas adelante seguimos con la discusión y aportes del Posibilismo. 
En este sentido se advierte la importancia del "posibilismo", proyectado en las primeras décadas del siglo veinte, reconocido como una "verdadera teoría general de las relaciones entre al hombre y la naturaleza; contrapuesto al determinismo alemán y al posibilismo radical (basado en lo social).

El posibilismo, teóricamente propuso comprender como objeto de estudio a la "elección que hace [el hombre] entre las diversas posibilidades presentes en su medio, y las estrategias socio-ecológicas que elabora no implican el que su libertad sea total" (Ib.: 7). En este sentido, la libertad se manifiesta en la forma que tiene el hombre para integrar su experiencia en esquemas de interpretación, creatividad que adquiere por medio de la cultura.

Para la antropología el posibilismo aportó el conocimiento de los hechos <contingentes>. En este sentido, la contingencia constituye la individualidad, entendida como resultante de las intersecciones de <series causales independientes>, hechos contingentes, mismos que integrados a los análisis culturales permiten comprender la existencia del método, modelo explicativo, basado en la causalidad del fenómeno, en la mayoría de los casos con intencionalidad humana.

El posibilismo vidaliano, privilegió como objeto de investigación la interacción entre el hombre y el medio, acercó a los estudios antropológicos de carácter estructural, los cuales plantearon el acercamiento con la variabilidad de códigos culturales. Esta corriente, permitió considerar al hombre como parte de la naturaleza, idea trascendente para la comprensión de los estudios sobre modos de vida.

Sin dejar de lado que existieron otras propuestas de carácter sociológico para el estudio del espacio, y mientras que unos se alinearon a la propuesta alemana y desarrollaron la geografía regional otros lo hicieron a la francesa, desarrollando la geografía cultural.

Por otro lado, resulta importante la integración de la sociografía ${ }^{6}$ entendida como ciencia que estudia la morfología social, categoría propuesta por Marcel Mauss, ${ }^{7}$ como producto de la conjunción entre la demografía y la antropogeografía; siendo que para él la polémica entre lo estrictamente sociológico (demográfico). y la geográfico es perfectamente inútil y su conjunción es "sólo cuestión de tiempo". La polémica generada, por pretender acceder a lo social a través de lo concreto, generó los indicadores necesarios para definir: edad, sexo, estado civil, ingresos, pero no solucionó el problema planteado para la comprensión de la interacción hombre-naturaleza ${ }^{8}$.

En consecuencia, se hizo necesaria una ciencia natural pura que estudiará los fenómenos humanos en su dependencia de la naturaleza, una geografía que tratará al hombre como ser social, explicar por todos los medios, las relaciones sociales de un pueblo en un tiempo determinado; la sociografía, particularmente la holandesa defendió está necesidad.

Contemplando la imposibilidad de acceder a la estructura interna de la sociedad a través sólo del paisaje se generó un debate no concluido, mismo que dejó a las disciplinas diferen-

6 En Holanda R. Steinmetz catedrático de la universidad de Amsterdam, con base en su experiencia en geografía física y humana, fue quien "acuñó el término de sociografía para designar la disciplina que se ocupa del estudio de los pueblos y de sus partes (ciudades, comunidades, etc.). en su peculiaridad o especificidad, en esencia es la descripción de la sociedad, con base en la experiencia humana. Esta sociografía suministraría material a la sociología -ciencia en exceso teórica en su opinión-, debiendo sustituir a la geografía regional" (Luis Gómez, 1983). 7 "Designamos con esta palabra la ciencia que estudia, con objeto de describirlo y explicarlo, el sustrato material de las sociedades, es decir, las formas que adoptan al establecerse en algún lugar, el volumen y la densidad de población, la forma en que ésta se distribuye y además todas aquellas cosas que sirven de base a la vida colectiva" (Mauss, 1971: 360).

8 "La situación propiamente geográfica está lejos de ser el hecho esencial sobre el que hay que centrar nuestra atención, pues es sólo una de las condiciones de las que depende la forma material de las agrupaciones humanas [...] En una palabra el factor telúrico ha de ponerse en contacto con el medio social en su totalidad y complejidad; no puede quedar aislado" (Ib.: 363). 
tes tareas, el campo de lo social a las ciencias sociales: a los sociólogos, a los etnólogos y antropólogos, a los historiadores, incluso.

\section{Geografía Cultural}

Disciplina que a decir de autores como López Levi, se puede definir como el estudio de las prácticas de vida y de expresión que tiene el ser humano. En su desarrollo histórico ubicamos tres periodos: la escuela norteamericana de Berkeley, la geografía humanista y la nueva geografía cultural.

En síntesis, la geografía cultural, propugnada por la escuela norteamericana de Berkeley, conjugó los amplios conocimientos de antropólogos y geógrafos alemanes: Boas, Kroeber y Lowie, especialmente en la obra de Carl Sauer, para quien la geografía debía de huir de posiciones nomotéticas y concentrarse en las diferencias espaciales. Sauer se centró en aspectos culturales para el análisis de los efectos que sobre el paisaje provocaron los distintos grupos sociales que lo habitaron. ${ }^{9}$

En este principio, desde la perspectiva de Sauer el objetivo de la geografía sería el paisaje, además de que para él "la cultura era el agente; el espacio natural, el medio y el paisaje cultural, el resultado”(López Levi, 2003).

"El objetivo de la escuela saueriana es por tanto la reconstrucción histórica del medio natural y de las fuerzas humanas que modifican el paisaje, la identificación de regiones culturales homogéneas definidas en base a elementos materiales (cerámica, material de construcción o tipos de viviendas). o bien elementos no materiales como religión o lenguas y dialectos; y por último el estudio de la ecología cultural histórica presentando especial atención en como la percepción y uso del paisaje viene condicionada por elementos culturales" (Luna García, 1999).

De acuerdo con el autor, para la presente investigación se retomó la idea de que la percepción y uso del paisaje son condicionadas por la cultura, puesto que las transformaciones que se observan en el entorno de las ciudades determinan el paisaje urbano que se erige, aunado a los acontecimientos sociales que también contribuyen a su transformación y provocan movilidad en los distintos sectores sociales.

De antemano, se reconoce que la propuesta de la geografía cultural, fue al tiempo impactada por los planteamientos de la sociología e historia urbana elaborados por Manuel Castells y Henri Lefebvre.

La geografía cultural tradicional, más el impacto de los marxistas contribuyeron a enriquecer las categorías de espacio, medio y paisaje con conceptos como identidad, significado e imaginación.

Producto de la divergencia, otros geógrafos críticos de la escuela de Berkeley, se acercaron a los estudios culturales, reconociéndose como geógrafos humanistas. Su acercamiento teórico retomó ideas de la sicología, la filosofía, específicamente de la fenomenología y el existencialismo, resultado de esto es la geografía de la percepción donde se analiza desde la perspectiva de la población, en relación a temáticas como los desastres el medio ambiente y el espacio cotidiano. ${ }^{10}$

Adicionalmente, a las aportaciones anteriores se agregan los presupuestos teóricos, de la ecología cultural, la cual se interesó por investigar en las formas del cómo vive la gente,

9 Las diferentes propuestas elaboradas por la escuela de Berkely y C. Sauer se encuentra mencionadas en la colección de artículos presentados en la revista («Documents d’Anàlisi Geogràfica 34», 1999)..

10 López Levi, subrayó el uso de mapas mentales, en esta corriente teórica de la geografía: "a fines de los años setenta y principios de los ochenta, autores como Cosgrove, Ley y Jackson, [...]rescataron la tradición humanista por encima de enfoque ambiental que caracterizaba a la Escuela de Berkeley" (2003:196). 
en sus actividades, en la calidad de sus vidas, por el tiempo que permanecen haciendo lo mismo y por las limitaciones sociales y medioambientales que deben superar (Luna García, 1999).

Determinantes antecedentes teóricos, tuvieron un alto impacto en los enfoques multidisciplinarios como el de la escuela de ecología cultural que se mueve entre la geografía, la sociología y la antropología, con el objetivo de comprender la relación existente entre los seres humanos, los recursos naturales y el espacio.

Derivados de las discusiones sobre el espacio entre geógrafos, historiadores, sociólogos y antropólogos, conjuntamente con la importancia que la geografía cultural tuvo después de la Segunda Guerra Mundial, se comprendió la necesidad de integrar una serie de conceptos que definieran el objeto de estudio de estas disciplinas y que acercaran al comprensión de la acción del ser humano sobre la naturaleza y el espacio.

La construcción teórica entre la geografía física y la geografía del paisaje, con enfoque funcional, contribuyeron a la explicación de las áreas espaciales homogéneas y los espacios heterogéneos, se comprendió que existía una unidad interna debida a la acción del ser humano: los espacios funcionales antropógenos. ${ }^{11}$ Espacios heterogéneos que tienen una unidad interna, homogénea, debida a la acción de las fuerzas propias de la sociedad, podían no estar continuos sino distantes entre sí.

Esta categoría permitió la clasificación de los espacios, la cual integró variables de carácter económico, étnico y cultural, que fueron retomadas por geógrafos como Waibel ${ }^{12}$, para diferenciar a los grupos sociales en referencia a su configuración del espacio. Se sobre entiende que el esfuerzo teórico se construyó para comprender cómo la estructura externa de un paisaje se conecta con la acción de la fuerzas sociales responsables de su fisonomía. ${ }^{13}$

Al respecto de la comprensión del dominio del espacio, Ratzel en su antropogeografía formuló el concepto de territorio, entendido como la porción de superficie terrestre apropiada por un grupo humano, y el concepto de espacio vital, territorio de una determinada sociedad transformado según sus recursos demográficos, tecnológicos y naturales disponibles. En este sentido, el territorio es objeto de la historia, dada la importancia que como espacio vital genera para la sociedad y el Estado.

De la conjunción anterior, el Estado conforma su dominio sobre el espacio; "la preservación y ampliación del espacio vital se convierte [...] en la razón de ser del Estado" (Lobato Correa, 1998a: 23).

Líneas arriba se mencionó la concepción del espacio absoluto y del espacio relativo, es pertinente retomar la concepción propuesta por Lefebvre, quien relacionó el espacio social, vivido, con la práctica social, distinta del espacio absoluto: "El espacio no es el punto de partida (absoluto). ni el punto de llegada (espacio como punto social). [...] tampoco es un instrumento político. Para Lefebvre, el espacio conjuntaba estas concepciones, pero es más que esto, "es el locus de la reproducción de las relaciones sociales de producción". Ni objeto, ni suma de objetos (Lefebvre, 1976).

Desprendida de la anterior, se encuentra la concepción de formación socioespacial, elaborada por Milton Santos, la cual presenta el espacio como un factor social y no como un reflejo social "el espacio se constituye en una instancia de la sociedad" (Id.). y (Santos, 1996).

Para Santos la noción gira en torno al espacio organizado por el hombre como una estructura subordinada-subordinante; espacio/sujeto/cultura en acción organizada sobre el

11 Llamados así por el Ratzel en su antropogeografía.

12 Waibel, estudio las praderas, los cafetales y las pequeñas unidades campesinas en la Sierra Madre de Chiapas (Citado en Luis Gómez, 1983: 24).

$13 \mathrm{El}$ concepto de antropógeno fue utilizado por la geografía urbana. Ver: Los lugares centrales en el sur de Alemania, Tesis doctoral de Walter Christaller, geógrafo austriaco que proponía el modelo de lugares centrales en 1933 (su texto fue traducido al inglés en 1966).(Christaller, 1933). 
medio. El autor propone comprender la categoría de espacio a partir del análisis de los términos: función, forma, estructura y proceso (Lobato Correa, 1998: 32).

La forma es el espacio visible, exterior, de un objeto, según se vea de manera aislada, o considerando la disposición de un conjunto de objetos, constituyendo un patrón espacial: una casa, un barrio, una ciudad; son conjuntos espaciales a diferentes escalas:

La función, actividad o papel desempeñado por el objeto creado por la forma. Son por ejemplo, la acción de habitar, vivir lo cotidiano, en sus múltiples dimensiones: trabajo, comprar, descansar, etcétera.

Estructura, establece a la naturaleza social y económica de una sociedad en un momento dado en el tiempo: es la matriz social donde las formas y funciones son creadas y justificadas.

Proceso, definido como una acción que se realiza como regla, de modo continuo, viendo y analizando un resultado cualquiera, implicando tiempo y cambio. Los procesos ocurren en el ámbito de una estructura social y económica y resultante de las contradicciones internas de las mismas. El proceso es una estructura en su movimiento de transformación.

La conjunción de estos conceptos conforma el paradigma, que para el análisis del espacio formuló M. Santos. De igual forma, permitió comprender en su totalidad el contexto espacial de la vida cotidiana, la imagen del espacio en lugares conformados por la sociedad. Además, se logró comprender la influencia del territorio sobre la identidad sociocultural.

En relación a lo anterior se retoma la discusión en torno al paisaje, la región y el espacio; discusiones y aportaciones que al respecto de la geografía cultural, se realizaron en las escuelas: alemana, francesa y norteamericana.

El paisaje cultural, en la geografía alemana

Como resultado de las propuestas alemanas que pretendían la separación entre la geografía física y la humana, se desarrolló el concepto de paisaje cultural.

Para los alemanes Schlüter ${ }^{14}$ y Hettner, que consideraban a la geografía solamente como una morfología de las formas de la superficie terrestre, Richthofen convino en afirmar que debería ocuparse de las relaciones existentes entre el hombre y la naturaleza, problema de método que en principio se resolvió con la descripción del paisaje, entendido desde una perspectiva netamente fisonómica, poco a poco se centró en el debate, como un concepto fundamental de la geografía, sobre manera cuando enfrentaron a la descripción de los grupos humanos. Con este objetivo la geografía se convirtió en una morfología del paisaje cultural, las preguntas de investigación se derivaron hacia el cómo los grupos humanos lo producen a lo largo de la historia y cómo esto ha sido y es determinante en el paisaje cultural, por lo que se le interpretó como un todo que integró el aspecto morfológico y estructural. Propuesta novedosa que conllevo la búsqueda de métodos para su abordaje.

La respuesta se encontró en las descripciones cuasi naturalistas desprendidas del trabajo de campo, las cuales se enfrentaron a la enorme dificultad para integrar la organización espacial de las sociedades contemporáneas.

Los trabajos referidos a la morfología del paisaje cultural, situaron a la geografía en el enfoque regional ${ }^{15}$. El estudio en diversas escalas hizo de la geografía una ciencia encar-

14 Ver nota núm. 60 en (Luis Gómez, 1983). [...] Schlüter exigía, a la hora de definir la ciencia geográfica, una «limitación en el objeto y, simultáneamente, total libertad en el enfoque». Dado que, en su opinión, sólo podían diferenciarse objetos, y como esto era de vital importancia para el mantenimiento de la ciencia geográfica como disciplina autónoma, nuestro autor indicaba que «en la antropogeografía falta, por lo tanto, la parte descriptiva, la morfología»,

15 Hettner en 1927, distinguió las siguientes escalas: continental, nacional, regional y local, en su geografía regional comparativa (Luis Gómez, 1983). 
gada de la descripción de las regiones, para la cual Hettner propuso la geografía regional comparativa.

La concepción anterior, reafirmó su rechazó a los fisonomistas, y procedió con cautela en relación con las nociones de paisaje cultural el cual "[...] debería considerar al ser humano no sólo como un adorno en el paisaje, sino también como un pedazo de su esencia" (Ib.: 11).

El pasaje cultural estableció la posibilidad de comprender la intervención del ser humano en la naturaleza, determinante de la configuración incluso física de la Tierra; tal es el caso de las villas, provincias, ciudades, etcétera. Sin embargo faltan los cambios que se perciben con respecto al tiempo, sus ritmos y ciclos que también se producen en la naturaleza y en los espacios urbanos, comprendidos como antropógenos puesto que funcionan en relación a las posibilidades de creación humana. Posición que de manera crítica y complementaria retomará la geografía cultural norteamericana.

\section{Geografía Humana Francesa: El Modo de Vida, el Espacio y la Organización Espa- cial de la Sociedad}

La geografía social francesa contribuyó al desarrollo teórico de la disciplina con la conformación de un cuerpo de conceptos que con base en la crítica al determinismo ratzeliano, el posibilismo y las propuestas de la ecología cultural, centraron su interés en el modo de vida más que en el paisaje cultural (Herin, 1982).

Por otra parte, se reconoce la influencia de los historiadores en las investigaciones geográficas. Las cuales dieron una ordenación dinámica a las investigaciones realizadas sobre los paisajes y las estructuras sociales, ampliando sus estudios al paisaje urbano:

"La ambición de estas investigaciones es comprender los paisajes, sus diferenciaciones y sus dinámicas, para lo cual hacen intervenir las estructuras sociales como factor esencial de explicación, sin que se recurra a grandes hipótesis referentes a la evolución económica, social y cultural de las sociedades rurales o urbanas" $(I b .: 5)$.

De esta ambición se generó la llamada geografía sociológica, que tuvo como objetivo el estudio de las clases sociales y los modos de vida, individual y social, lo que permitió una geografía social por clases y grupos: los obreros, la burguesía, el clero, y otros. decir:

Con el apoyo del método comparativo se creó la geografía sociológica comparada, es

"Una geografía que, procediendo por superposición de los mapas de los diferentes comportamientos y de la distribución espacial de las clases sociales y de las características de la vida social, permitirá poner en relación (en correlación)., por concordancias y diferencias, las diversas maneras de ser, de pensar y de comportarse" (Ib. 6). ${ }^{16}$

Para la comprensión de los paisajes culturales, se requirió de la historia además de la mirada del sociólogo, que combinadas con los métodos de observación directa del geógrafo permitieron encontrar el sentido a las descripciones del espacio geográfico y derivar de la sociología, el espacio social; a saber, el espacio de las interrelaciones con los grupos sociales y con los lugares.

La dimensión sociológica dejo a la geografía, al margen de las descripciones morfológi-

16 Visión que se ejemplifica en las investigaciones de F. Braudel sobre el Mediterráneo y sobre la identidad en Francia. 
cas, su acercamiento a una geografía del espacio vivido ${ }^{17}$; de las relaciones que unen a los hombres a sus lugares, a los paisajes, al espacio (Herin, 1982).

Mediante el análisis del espacio vivido la geografía se da así por objeto el describir e interpretar las relaciones espacio-sociales que unen a los individuos, los grupos sociales y los diferentes niveles del espacio ( $I b .:$ 9). Estas relaciones son múltiples:

Relaciones de producción: por ejemplo, el espacio rural es principalmente el espacio de la producción agrícola; en este se localizan las producciones agrarias y ganaderas. Para las labores agrarias, por ejemplo, los trabajadores y productores campesinos, mantienen con el espacio relaciones complejas, para ellos el valor del espacio radica en su capacidad de producción.

Relaciones de hábitat: la densidad de la población, la frecuencia de los habitantes, sus distribuciones a diferentes escalas, su carácter permanente o temporal, etc. Todo ello señala y mide la presencia y el control desigual de los hombres sobre el espacio mismo que producen, habitan y significan.

Relaciones de desplazamientos, de frecuencia y de distancia variada; por la producción y los intercambios, o por las relaciones sociales no económicas tejen, entre los individuos y los lugares, integran las redes cuantificables en cuanto a intensidad y distancia, que alimentan las correspondencias difíciles de captar.

Relaciones del tiempo libre bajo sus formas diversas: movilidad, consumo, vivienda temporal o secundaria, descubrimiento de paisajes, ocio, etc. (Ib.:12). El estudio de las relaciones se enlaza con el de los grupos sociales, pero en el entendido de que se realizan en escalas diferentes, mismas que emplazan los lugares. Para lo cual, es pertinente indicar las definiciones que para los conceptos de lugar, paisaje y espacio, que de manera general distingue la geografía social francesa:

Lugar: se refiere a la escala de lo local, expresada en toda la complejidad concreta de las interrelaciones que lo constituyen;

Paisaje (geográfico). se comprende más bien la disposición tangible, visible, palpable de las relaciones entre el medio natural y los grupos sociales, lo cual, en un sentido amplio, no supone necesariamente cohesión y coherencia;

Espacio, concepto abstracto, comprende a la vez los aspectos tangibles del paisaje geográfico y las dimensiones ocultas que están subyacentes, lo estructurante y lo explicativo, cuya revelación es la meta de la investigación.

Producto de las investigaciones sobre las relaciones geográficas se sabe que construyen relaciones con el espacio: de vinculación, por ejemplo las actividades de producción y de habitar; las de adaptación, relaciones ecológicas que determinan la ordenación del espacio. Con respecto al tiempo y tomando en cuenta que el paisaje conlleva la huella y el testimonio de sociedades anteriores, se construyen relaciones espaciales de carácter histórico. En este sentido, se reconocen relaciones, vínculos de apego y arraigo que crean lazos afectivos al espacio positivo e incluso negativo.

Con base en estas relaciones y otras, se sabe que algunos lugares tienen una función simbólica e ideológica valorada por el grupo social que lo usa y práctica, por ejemplo: "lugares de culto y de peregrinación, monumentos civiles y militares. Para las multitudes que se congregan, el lugar tiene un valor de símbolo mediador de la fe o de las convicciones que se expresan $(I b .: 13)$.

17“El espacio vivido es una experiencia continua, egocéntrica y social, un espacio de movimiento y un espacio de tiempo... [que] se refiere a lo afectivo, a lo mágico, a lo imaginario. Holzer, 1992, citado por Lobato Correa (1998).. 
De acuerdo con esta construcción espacial de los lugares, mismos que pueden tener una función simbólica, expresada en las relaciones, las prácticas percepciones del espacio, se deriva que estas se relacionan con la producción, la economía, la política, la religión y la cultura en un sentido extenso.

Robert Herin, mostró que las prácticas del espacio permanecen dependientes de la posición de clase, que unida a la comprensión de las escalas permite mayor comprensión del significado social de los espacios, tanto en áreas rurales como urbanas.

Desde la perspectiva conceptual de Herin, subrayó que como herencia de la geografía alemana, la geografía francesa vidaliana tiene por objetivo el estudio de los grupos humanos en su relación con el medio que le rodea, lo cual determina su modo de vida. Esta perspectiva se relaciona con el trabajo de los antropólogos quienes describen, ubican a los sujetos, grupos sociales y sus relaciones, vinculación directa con otro de los objetivos de la geografía cultural explicar la organización espacial de la sociedad.

\section{Región cultural}

En cuanto al concepto de región y de región cultural, se subraya que surge de manera histórica, pero para la discusión que nos ocupa convino su importante vinculación con la región natural.

En cuanto a la región desde el lenguaje cotidiano tiene relación con el principio de localización y el de extensión. Paulo C. Da Costa G. señaló su empleo como "una referencia asociada a la localización y a la extensión de cierto hecho o fenómeno, o incluso ser una referencia asociada a límites más o menos habituales atribuidos a la diversidad espacial" [...] donde predominan determinadas características que distinguen aquella área de las demás (Da Costa Gomes, 1998).

Si bien, es conocida su acepción como unidad administrativa, o asociada a la localización de cierto dominio, la región existe históricamente, como la referencia a las tradiciones culturales, por ejemplo las egipcias, romanas o griegas; en consecuencia es una referencia a la región natural que domina o influencia a la configuración de una sociedad.

En relación a lo anterior, es evidente que el concepto de región natural se corresponda con la idea de ambiente; por lo cual se encuentra en el centro de los debates epistemológicos de la geografía.

Para el 'posibilismo', la región existe como unidad básica del saber geográfico, el cual es resultado del trabajo humano en determinado ambiente. Noción que en la tradición alemana y francesa generó una amplia bibliografía sobre monografías de diversas regiones geográficas. Más aún, con la perspectiva culturalista francesa, se desarrolló una metodología:

"Se debe empezar por la descripción de las características físicas, seguida de la descripción de la estructura de la población y de sus actividades económicas. El objetivo final es encontrar en cada región una personalidad, una forma de ser diferente y particular [su morfología] o cualquier elemento a partir del cual una comunidad territorial crea una forma diversa de adaptarse" (Ib.: 53).

La región, en el centro del debate entre la geografía clásica cultural y la nueva geografía, la empirista descriptiva de la relación hombre/medio. Además de la cientificista, que pretende que la región sea una técnica, un medio para la demostración y no un producto más de la investigación. Al respecto, advirtió Da Costa que en esta perspectiva surgieron dos tipos de regiones: las homogéneas y las funcionales o polarizadas. Las primeras definen espacios más o menos similares; en cuanto a la segundas, la referencia es la las múltiples relaciones que circulan y dan forma al espacio que al interior es diferenciado. 
Dentro de los estudios que sobre las regiones se realizaron, Da Costa subrayó tres debates: la primera discusión se dio con respecto a la región natural frente a la región geográfica. En el centro de este debate se encuentra el modelo explicativo, el cual indica como causa directa a las condiciones naturales, para entender e interpretar la diversidad en la organización social. Asimismo, analizó la relación entre sociedad y ambiente, correspondencia dinámica en la que hay reciprocidad, a decir de Vidal de la Blache. Otra cuestión se dio acerca de la región, discutida entre los modelos de la ciencia de lo general y de una ciencia de lo particular. "En el primer caso el modelo es analítico y se destina a producir leyes generales y medidas objetivas en la observación de los hechos estudiados. [...] la región es vista como el resultado de una clasificación, un tipo de área obtenida a través de un criterio analítico de extensión espacial". Mientras que en el modelo sintético, el trabajo intelectual no se elabora a partir de ideas, conceptos abstractos, producidos por generalizaciones; sino a partir, de categorías que se definen por la descripción de casos concretos. Desde esta perspectiva la región se concibe como una realidad autoevidente, físicamente construida, sus límites son permanentes y definen un marco de referencia fijo percibido mucho más por el sentimiento de identidad y pertenencia, que por la lógica ${ }^{18}$.

Por último, la discusión que pretende identificar entre criterios generales y uniformes que estructuran el espacio con criterios mutables que se definen por la dirección de la explicación. Las regiones son así, en el primer caso, el resultado de una división del espacio que en principio es sometido siempre a las mismas variables, definiéndose así a través de esta división un sistema espacial clasificador, uniforme y jerárquico. En el segundo caso, las regiones son concebidas como productos relativos, fruto de la aplicación de criterios particulares, tienen un carácter demostrativo en la comprensión del dominio de ciertas variables al interior de determinados fenómenos.

Muchos autores han señalado la importancia de las regiones, concepto que implica la revisión histórica, antropológica, sociológica y geográfica; si bien, en la intensa homogenización que se vive a través de los procesos de globalización. Las regiones están "envueltas en una dinámica cultural hegemónica", mientras que, aún se generan movimientos de resistencia que han mantenido "el derecho a la diferencia". Los debates en torno a las regiones y la manera de abordarlas continúan e implican una mayor reflexión, discusiones sobre sus límites, su capacidad de resistencia, la forma en que enfrentan al capitalismo, al poder; manifiestan que las regiones se mantienen por la comunidad que las contiene, los procesos culturales, de identificación, de autonomía que se viven en su interior, además de las relaciones que generan con el vasto mundo.

\section{Espacio funcional antropógeno urbano}

A manera de reflexión final, se recuperaron las aportaciones de la geografía humana y cultural que se consideran necesarias para el estudio del espacio desde la perspectiva de la antropología. En cuanto a la antropogeografía, y su llamado a la interdisciplinariedad en las ciencias sociales; teóricamente concibió el espacio como necesario e indispensable en la vida del hombre. En consecuencia propuso dos conceptos clave territorio y espacio vital, siendo que en el primero se condensa la apropiación de un espacio por determinado grupo, mientras que en el espacio vital, se comprende la necesidad de la sociedad en relación con la tecnología, la densidad de población y la relación que se construye con la naturaleza (recursos naturales). Si el espacio vital y el territorio, permiten emplazar el estudio de la relación de la sociedad con su entorno, también lo es la relación entre el espacio y el tiempo; las cuales conjuntan la dimensión sociocultural y de vida de los sujetos. En este orden, es posible comprender en el espacio, el estudio de los fenómenos desde una perspectiva 
histórico-cultural, en donde la dimensión temporal permite observar y analizar las formas de apropiación, y relación social.

En consecuencia, se asume a la manera de Harvey, que el espacio "no es en sí mismo ni absoluto, ni relativo, ni relacional, pero puede llegar a ser una de estas cosas o toda a la vez según las circunstancias" (Harvey, 1977). En tanto que, conceptualizar el espacio significa hablar de la práctica humana en él.

En este caso, la propuesta comprende la experiencia sensible, cinestésica, misma que se obtiene mediante el uso de dibujos, croquis y/o mapas mentales, que registran la práctica del espacio junto con la experiencia del sujeto puesto que relaciona lo sensible por la práctica a través de la memoria, (pensamiento/cosmovisión). cultural, conjunción que permite señalar que la experiencia espacial, genera el espacio simbólico.

En este sentido, se considera que las aportaciones de la geografía al conocimiento antropológico e incluso a las ciencias sociales, permiten reconocer que el espacio no es sólo un contenedor de las relaciones sociales, sino que "la sociedad se torna concreta a través del espacio, del espacio que ella produce y, por otro lado el espacio únicamente es inteligible a través de la sociedad" (Lobato Correa, 1998a: 30).

La historia y etnicidad de los hombres, se asume, toma sentido cuando se emplazan en su tiempo y espacio, que de manera dialéctica actúan para determinar las características culturales de los diferentes grupos sociales que forman la sociedad. Por lo que los estudios geográficos y antropológicos se completan cuando se comprende la relación dialéctica que construye el hombre con su medio.

Otra aportación tiene relación con la construcción teórica, con enfoque funcional; la geografía física como la geografía del paisaje, contribuyeron a la explicación de las áreas espaciales homogéneas y los espacios heterogéneos, con el concepto de espacios funcionales antropógenos, espacios producto de la intervención humana, en este sentido la ciudad sería un ejemplo. Consecuentemente el espacio antropógeno genera paisajes culturales.

El espacio así definido, necesita para su comprensión de una mirada interdisciplinaria que combine los métodos de observación tanto del geógrafo como del sociólogo, en un proceso de interpretación que implica la mirada culturalista del antropólogo.

La conjunción del espacio funcional antropógeno urbano, empata con la propuesta de Lefebvre que relacionó el espacio absoluto con el espacio social, vivido, comprendiendo el espacio como el lugar de la reproducción de las relaciones sociales. Lefebvre, generó indirectamente un objeto a la geografía cultural: el análisis del espacio vivido.

La geografía nos acerca al espacio vivido, mientras que la sociología al espacio social, ambas se complementan cuando se discute sobre las relaciones que unen a los hombres, a sus lugares, conformando paisajes culturales que se fomentan en el escenario urbano.

\section{Bibliografía}

BERDOULAY, V.

1983 "Perspectivas actuales del posibilismo". Geo Crítica, Cuadernos críticos de Geografía Humana, Año VIII, Núm. 47. Recuperado 5 de junio de 2014, a partir de http://www. ub.edu/geocrit/geo47.htm

CASTRO, I. E. DE, GOMES, P. C. DA C., \& CORRÊA, R. L.

1995 Geografia: conceitos e temas. Rio de Janeiro: Bertrand Brasil.

CASTRO, I. E. DE, GOMES, P. C. DA C., \& CORRÊA, R. L. (Edits.)

1996 Brasil: questões atuais da reorganização do território. Rio de Janeiro: Bertrand Brasil. CHRISTALLER, W.

1933 Los lugares centrales en el sur de Alemania. Erlangen: Universidad de Erlangen, Alemania.

DA COSTA GOMES, P. C.

1998 “El concepto de región y su discusión”. En ¿Cómo pensar la Geografía? Cuadernos de 
Geografía Brasileña (Vol. 1, pp. 47-68). México: Centro de Investigación Científica «Ing. Jorge L. Tamayo», A.C.

DELGADO MAHECHA, O.

2003 Debates sobre el espacio en la geografía contemporánea. Bogotá: Universidad Nacional de Colombia.

\section{HARVEY, D.}

1977 Urbanismo y desigualdad social. Madrid: Siglo XXI.

HERIN, R.

1982 "Herencias y perspectivas en la geografía social francesa". Geo Crítica, Cuadernos críticos de Geografía Humana, Año VI. Núm. 41. Recuperado 5 de junio de 2014, a partir de http://www.ub.edu/geocrit/geo41.htm

\section{LEFEBVRE, $\mathrm{H}$.}

1976 Espacio y política: el derecho a la ciudad II. Barcelona: Península.

LOBATO CORREA, R.

1998a "Espacio, un concepto clave de la geografía". En Cuadernos de Geografía Brasileña (pp. 21-46). México: Centro de Investigación Científica «Ing. L. Tamayo», A. C.

\section{LOBATO CORREA, R.}

1998b "La dimensión cultural del espacio: Algunos temas".¿Cómo pensar la Geografía? Cuadernos de Geografía Brasileña (Vol. 1, p. 105.114). México: Centro de Investigación Cien-

LÓPEZ LEVI, L. tífica «Ing. Jorge L. Tamayo», A.C.

2003 "Geografía cultural y posmodernidad: nuevas realidades, nuevas metodologías". En Oliver, Patricia (Edit.). Espacio, geográfico, epistemología y diversidad. México: UNAM.

LUIS GÓMEZ, A. L.

1983 "La Geografía Humana: ¿De ciencia de los lugares a ciencia social?”. Geo Crítica, Año VIII, No. 48. Recuperado 5 de junio de 2014, a partir de http://www.ub.edu/geocrit/geo48. htm

LUNA GARCÍA, A.

1999 “Qué hay de nuevo en la geografía cultural?”. Documents d’Anàlisi Geogràfica, 34, 6980.

MAUSS, M.

1971 Sociología y antropología. Madrid: Tecnos.

MORLAINS, M. C.

2005 "Introducción a la ecología del paisaje”. Área Ecología, 1-33.

OLIVERA, P. E. (Edit.)

2003 Espacio geográfico, epistemología y diversidad. México: UNAM.

ORTEGA URIBE, G. (Comp.)

1998 ¿Cómo pensar la Geografía? Cuadernos de Geografía Brasileña. México: Centro de In-

PARK, R. E. vestigación Científica «Ing. Jorge L. Tamayo», A. C.

1999 La ciudad y otros ensayos de ecología urbana. Barcelona: Serbal.

ROMERO, C. M., \& Morláns, M. C.

s. f. "Evolución de la fragmentación del paisaje en el valle central de Catamarca, periodo 1973-2007”. Recuperado 5 de junio de 2014, a partir de http://www.editorial.unca.edu.ar/ ROSENBERG, $\mathrm{M}$.

Publicacione\%20on\%20line/Ecologia/imagenes/pdf/Cap_1.pdf

2014 "Biography of American Geographer Carl O. Sauer". Recuperado 7 de junio de 2014, a SANTOS, $\mathrm{M}$. partir de http://geography.about.com/od/historyofgeography/a/carlsauer.htm

1996 Metamorfosis del espacio habitado. Barcelona: Oikos-Tau.

SERRANO, Karla

s. f. "Tratado de Geografía Humana". Recuperado 5 de junio de 2014, a partir de https://www. academia.edu/6725768/Tratado_de_Geografia_Humana_5 